Laser Chem. Vol. 7, pp. 297-304

Photocopying permitted by license only

(C) 1987 Harwood Academic Publishers GmbH

Printed in the United Kingdom

\title{
Photodissociation of $\mathrm{CH}_{2} \mathrm{ClCH}_{2} \mathrm{I}$ at $308 \mathrm{~nm}$
}

\author{
TIMOTHY K. MINTON,* GILBERT M. NATHANSON, $\ddagger$ and \\ YUAN T. LEE
}

Materials and Molecular Research Division, Lawrence Berkeley Laboratory and Department of Chemistry, University of California, Berkeley, CA 94720, USA

(Received August 25, 1986; accepted in final form December 8, 1986)

\begin{abstract}
The technique of photofragmentation translational spectroscopy has been used to study the photodissociation of $\mathrm{CH}_{2} \mathrm{ClCH}_{2} \mathrm{I}$ at an excitation wavelength of $308 \mathrm{~nm}$. The exclusive dissociation pathway is $\mathrm{C}-\mathrm{I}$ bond breakage with formation of $\mathrm{CH}_{2} \mathrm{ClCH}_{2}$ and $\mathrm{I}\left({ }^{2} P_{3 / 2}\right)$. The centre-of-mass translational energy distribution of the photofragments reveals that an average of about $50 \%$ of the excess energy appears in translation. The angular distribution of dissociation products with respect to the laser polarization indicates that the photodissociation process proceeds via a parallel transition-i.e., the transition moment must be nearly parallel to the C-I bond. Exclusive production of ground state $\mathrm{I}\left({ }^{2} P_{3 / 2}\right)$ with a parallel polarization dependence is unexpected based on the prevailing picture for alkyl iodide photodissociation.
\end{abstract}

KEY WORDS: Photofragmentation, photodissociation, $\mathrm{CH}_{2} \mathrm{ClCH}_{2} \mathrm{I}$.

\section{INTRODUCTION}

The photodissociation dynamics of $\mathrm{CH}_{2} \mathrm{ClCH}_{2} \mathrm{I}$ have been studied previously in our laboratory at 248 and $266 \mathrm{~nm},{ }^{1}$ where an $n \rightarrow \sigma^{*}$ absorption localized on the $\mathrm{C}-\mathrm{I}$ bond is expected to occur. The translational energy distributions and angular distributions show that two dissociation pathways exist, leading to formation of either ground or spin-orbit excited state iodine, and that both channels at both excitation wavelengths originate from a parallel transition-i.e., the transition moment must be nearly parallel to the C-I bond. Even

* Present address: Dept. of Chemistry, University of Illinois, Urbana, IL 61801. $\ddagger$ Miller Fellow. 
though the polarization dependence is the same at both wavelengths, the $\mathrm{I}\left({ }^{2} P_{1 / 2}\right) / \mathrm{I}\left({ }^{2} P_{3 / 2}\right)$ branching ratios differ significantly $(1.5$ at $248 \mathrm{~nm}$ and 3.0 at $266 \mathrm{~nm}$ ). These observations do not fully conform to the commonly employed quasi-diatomic model, ${ }^{2}$ which is an extension of Mulliken's description of the $n \rightarrow \sigma^{*}$ absorption band in the hydrogen halides, the interhalogens, and methyl iodide. ${ }^{3}$ This model predicts a parallel polarization dependence for the $\mathrm{I}\left({ }^{2} P_{1 / 2}\right)$ dissociation channel and a perpendicular dependence for $\mathrm{I}\left({ }^{2} P_{3 / 2}\right)$ formation. Consequently, the model provides no explanation for the different branching ratios. By investigating the photofragmentation of many iodoethanes at different wavelengths, we hope that some trends will emerge, leading to a better understanding of the $n \rightarrow \sigma^{*}$ continuum in haloethanes. Accordingly, we have extended the data on $\mathrm{CH}_{2} \mathrm{ClCH}_{2} \mathrm{I}$ to include a third excitation wavelength at the low energy (long wavelength) tail of the $n \rightarrow \sigma^{*}$ continuum. ${ }^{4}$

\section{EXPERIMENT}

The experimental apparatus and conditions were virtually identical to those described elsewhere, ${ }^{5}$ and we will only briefly describe them here. The apparatus has a rotatable molecular beam source with a fixed mass spectrometer detector. A $308 \mathrm{~nm}$ excimer laser beam, which was focused to a spot size of $3 \mathrm{~mm} \times 0.4 \mathrm{~mm}$, crossed the molecular beam perpendicularly to the plane of the molecular beam and detector. Time-of-flight (TOF) measurements of the photofragments were taken with two laser polarizations: horizontal and vertical parallel and perpendicular, respectively, to the detector axis). Average laser pulse energies were $9.9 \mathrm{~mJ}$ for horizontal polarization and $15.4 \mathrm{~mJ}$ for vertical polarization. This difference in pulse energies is a result of the method used to separate the two polarizations, which favored the transmission of the vertical component. Laser saturation was unimportant at these energies because the absorption cross section at $308 \mathrm{~nm}$ is very low $\left(\sim 3 \times 10^{-20} \mathrm{~cm}^{2}\right){ }^{4-6}$ The gas mixture for the molecular beam was formed by bubbling Ar through liquid $\mathrm{CH}_{2} \mathrm{ClCH}_{2} \mathrm{I}$, maintained at $20^{\circ} \mathrm{C}$ (vapor press. $=7$ Torr), to make a total stagnation pressure of 300 Torr. The mixture was allowed to expand through a $0.005 \mathrm{in} .(0.125 \mathrm{~mm})$ diameter nozzle, heated to $250^{\circ} \mathrm{C}$ to prevent dimer formation. Signal could only be detected at $\mathrm{m} / \mathrm{e}$ 
$=127\left(\mathrm{I}^{+}\right)$, and TOF data at this mass were taken at source angles of $20^{\circ}$ and $40^{\circ}$ (horizontal polarization) with count rates of 0.052 and 0.034 counts/laser pulse, respectively. One TOF was recorded with vertical laser polarization at $20^{\circ}$ with a count rate of 0.021 counts/pulse.

\section{RESULTS AND ANALYSIS}

The centre-of-mass (c.m.) translational energy distribution $P\left(E_{T}\right)$ and the c.m. angular distribution $w(\theta)$ for the photofragments are derived from our experimental, laboratory TOF distributions $N(t)$ and angular distributions $N(\Theta)$ by a forward convolution technique, where we assume that the c.m. flux distribution $I_{\mathrm{cm}}$ is given by the product, $I_{\mathrm{cm}}\left(E_{T}, \theta\right)=P\left(E_{T}\right) w(\theta)$. The form of the c.m. angular distribution is ${ }^{8}$

$$
w(\theta) \propto 1+\beta P_{2}(\cos \theta),
$$

where $\theta$ is the angle between the electric vector of the laser light and the c.m. recoil direction of the products. $\beta$ is the anisotropy parameter, which we derive, and it is equal to 2.0 for a purely parallel transition $\left(w(\theta) \propto \cos ^{2} \theta\right)$ and -1.0 for a purely perpendicular transition $\left(w(\theta) \propto \sin ^{2} \theta\right)$.

Figure 1 shows the TOF data, along with the best fits (using the $P\left(E_{T}\right)$ distribution in Figure 2), for the iodine fragment. Unlike the previous $\mathrm{CH}_{2} \mathrm{ClCH}_{2} \mathrm{I}$ experiments at 248 and $266 \mathrm{~nm},{ }^{1}$ these data do not exhibit two distinct components, suggesting that only one spin-orbit state of iodine is formed in the dissociation process.

We measured the angular dependence of the photofragmentation process by two methods, leading to two determinations of the anisotropy parameter. First, we compared the ratio of integrated signals from TOF's measured with horizontal and vertical laser polarizations (using a fixed source angle of $20^{\circ}$ ) to the ratios calculated for different $\beta$ values. Second, we compared the ratio of integrated signals from TOF's measured at $20^{\circ}$ and $40^{\circ}$ with a fixed horizontal laser polarization to the ratios calculated for various $\beta$ 's. The two best fit $\beta$ parameters are different; however, Table I shows that the horizontal/ vertical angular dependence is much more sensitive to $\beta$ than the $20^{\circ} / 40^{\circ}$ angular scan. This result is expected because $\cos ^{2} \theta_{\mathrm{cm}}$ changes significantly more between horizontal and vertical laser polarizations (at a source angle of $20^{\circ}$ ) than between source angles of $20^{\circ}$ and $40^{\circ}$ 


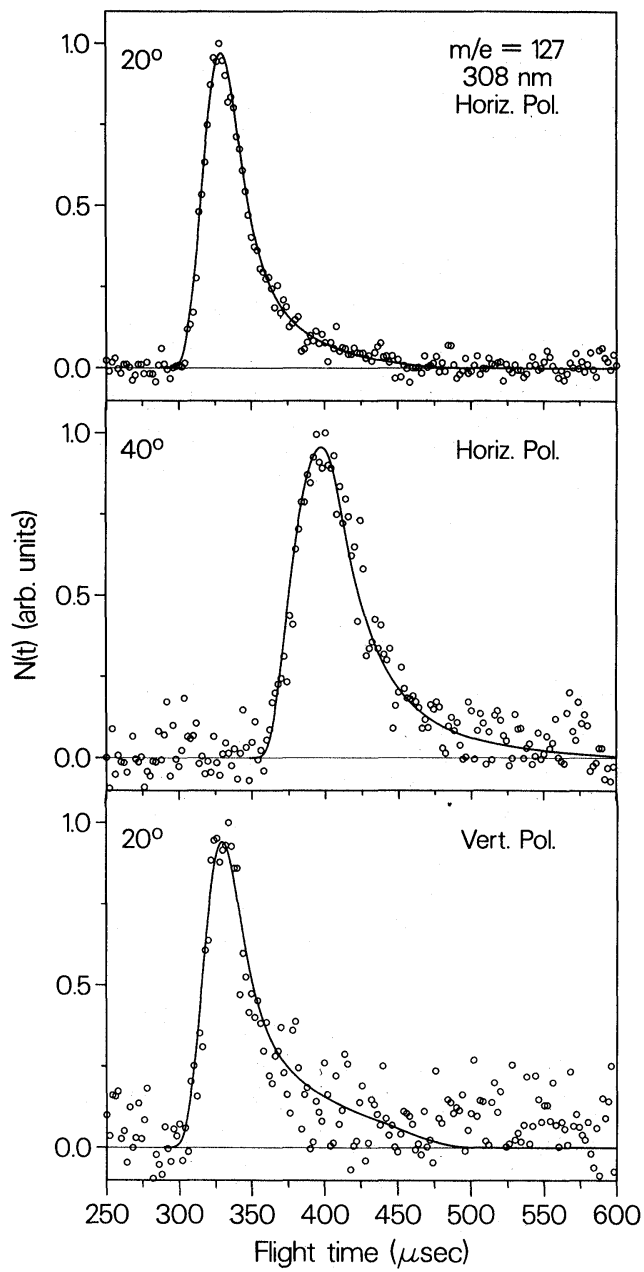

XBL 867-2708

Figure 1 Laboratory TOF distributions of I atom product. $\bigcirc$ Experimental points; calculated using the $P\left(E_{T}\right)$ in Figure 2 and $\beta=1.75$.

with fixed horizontal polarization. (A thorough discussion of anisotropy measurements is given in Ref. 5). Therefore, using only the more sensitive angular dependence, we will take as the value of the anisotropy paraments: $\beta=1.75 \pm 0.2$. The assigned error limits are a result of the uncertainty in the $P\left(E_{T}\right)$ distribution used in the calculation of $\beta$. 


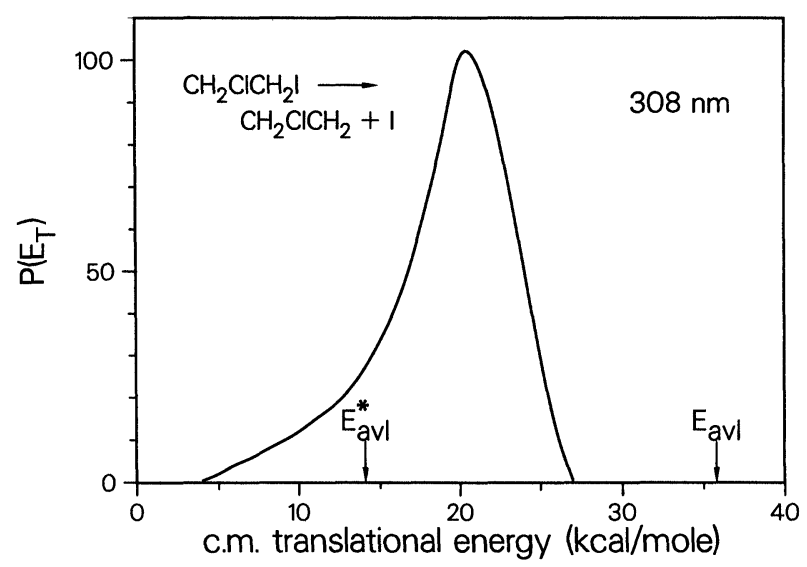

XBL $867-2706$

Figure 2 Center-of-mass recoil translational energy distribution for $\mathrm{CH}_{2} \mathrm{ClCH}_{2} \mathrm{I}$ photodissociation at $308 \mathrm{~nm}$. $E_{\text {avl }}$ is the excess energy after breaking the C-I bond in $\mathrm{CH}_{2} \mathrm{ClCH}_{2} \mathrm{I}$. $E_{\text {avl }}^{*}$ is $E_{\text {avl }}$ minus the I atom spin-orbit splitting $(21.7 \mathrm{kcal} / \mathrm{mole})$. Total fragment internal energy is given by $E_{\text {avl }}$ (or $E_{\text {avl }}^{*}$ ) minus the c.m. translational energy. Because the curve does not show evidence of two components and because the majority of the distribution is higher in energy than $E_{\mathrm{avl}}^{*}$, the exclusive dissociation channel must be $\mathrm{C}-\mathrm{I}$ fission with ground state $\mathrm{I}\left({ }^{2} P_{3 / 2}\right)$ formation.

The $\beta$ parameter and the fits to the TOF distributions were calculated from the translational energy distribution shown in Figure 2. The available translational energy for each of the two possible dissociation channels is indicated in the figure $\left(E_{\text {avl }}\right.$ for the ground state $\mathrm{I}\left({ }^{2} P_{3 / 2}\right)$ formation and $E_{\text {avl }}^{*}$ for excited state $I\left({ }^{2} P_{1 / 2}\right)$ formation). By energy conservation, most of the $P\left(E_{T}\right)$ distribution could only come from dissociation leading to ground state iodine product. Some part of the low energy tail might be caused by a small amount of $\mathrm{I}\left({ }^{2} P_{1 / 2}\right)$ formation, but we cannot determine the magnitude of this fraction or if it is, in fact, nonzero. Assuming the whole curve to be the result of $\mathrm{I}\left({ }^{2} P_{3 / 2}\right)$ formation, then the average energy in translation is 0.54 of the available energy. This fraction is very close to the usual observation for photodissociation of iodoethanes that about $50 \%$ of the available energy appears in translation. The energy range of the $P\left(E_{T}\right)$ distribution combined with the observation of only one peak in the time-offlight provides strong evidence that ground state iodine is the exclusive product. While we cannot rule out $\mathrm{I}\left({ }^{2} P_{1 / 2}\right)$ formation completely, its abundance must be small $(<6 \%)$, given the low $E_{\mathrm{avl}}^{*}$. 
Table I Observed and calculated signal ratios, using various values for the anisotropy parameter $\beta$, for horizontal vs. vertical polarization at a fixed source angle of $20^{\circ}$ and for $20^{\circ}$ vs. $40^{\circ}$ source angle with fixed horizontal polarization.

\begin{tabular}{llll}
\hline Trial $\beta$ & Horizontal/vertical & $20^{\circ} / 40^{\circ}$ & \\
\hline- & 4.00 & 1.59 & observed \\
1.00 & 2.31 & 1.52 & \\
1.10 & 2.48 & 1.56 & \\
1.20 & 2.68 & $1.59^{\mathrm{a}}$ & \\
1.30 & 2.88 & 1.63 & \\
1.40 & 3.10 & 1.67 & \\
1.50 & 3.34 & 1.70 & \\
1.60 & 3.60 & 1.74 & \\
1.70 & 3.88 & 1.78 & \\
1.75 & $4.03^{\mathrm{a}}$ & 1.79 & \\
1.80 & 4.18 & 1.81 & \\
1.90 & 4.51 & 1.84 & \\
2.00 & 4.87 & 1.87 & \\
\hline
\end{tabular}

${ }^{a}$ Best fit to $\beta$ for the respective ratio.

\section{DISCUSSION}

The most significant results from this study are the $P\left(E_{T}\right)$ distribution, which shows the overwhelming dominance of the ground state $\mathrm{I}\left({ }^{2} P_{3 / 2}\right)$ dissociation channel, and the anisotropy parameter, which is indicative of a parallel transition-that is, one in which the transition moment is parallel to the C-I bond.

The factors that affect $\beta$ are discussed in detail in Ref. 5, but it should be noted here that the fact that $\beta$ is less than 2.0 does not necessarily imply a combination of two types of transitions-parallel and perpendicular-or a transition moment which is not exactly parallel to the $\mathrm{C}-\mathrm{I}$ bond. The reduction of $\beta$ could be caused by distortion of the excited complex prior to dissociation, which would change the direction of fragment recoil with respect to the initial C-I bond axis. In any case, an anisotropy parameter of 1.75 (or even 1.2, the results from the less sensitive measurement) implies a predominantly parallel transition.

The observation of only $\mathrm{I}\left({ }^{2} P_{3 / 2}\right)$ formation via a parallel polarization dependence is surprising. Using a quasi-diatomic model, ${ }^{2}$ which assumes an $n \rightarrow \sigma^{*}$ transition localized on the C-I bond, we would predict that ground state iodine formation should originate from a 
perpendicular transition to the ${ }^{3} Q_{1}$ or ${ }^{1} Q$ state. A parallel transition to the ${ }^{3} Q_{0}$ state should correlate only toI $\left({ }^{2} P_{1 / 2}\right)$ product. Photodissociation of many alkyl iodides has been observed ${ }^{1,9-12}$ to produce both $\mathrm{I}\left({ }^{2} P_{1 / 2}\right)$ and $\mathrm{I}\left({ }^{2} P_{3 / 2}\right)$ products with the same, parallel polarization dependence, leading to the invocation of curve crossing to explain the results. This study of $\mathrm{CH}_{2} \mathrm{ClCH}_{2} \mathrm{I}$, however, marks the first observation of total ground state formation from a parallel transition. Within the context of the diatomic model, we would be forced to conclude that curve crossing occurs $100 \%$ of the time after an intial excitation to the ${ }^{3} Q_{0}$ state. But this explanation is unsatisfactory as curve crossing is not expected to be so efficient. The apparent failure of the simple picture suggests that a better understanding of the excitation process leading to dissociation would come from consideration of the molecule as a whole. A systematic study of the photofragmentation dynamics of many alkyl iodides in the $230-310 \mathrm{~nm}$ region would be an instructive link in the formulation of a successful model, which will describe the dissociation process in the $n \rightarrow \sigma^{*}$ continuum.

\section{Acknowledgement}

This work was supported by the Director, Office of Energy Research, Office of Basic Energy Sciences, Chemical Sciences Division of the U.S. Department of Energy under Contract No. DE-AC-0376SF00098. G.N. thanks the Miller Institute of the University of California for a fellowship.

\section{References}

1. T. K. Minton, P. Felder, R. J. Brudzynski and Y. T. Lee, J. Chem. Phys. 81, 1759 (1984).

2. D. Krajnovich, L. J. Butler and Y. T. Lee, J. Chem. Phys. 81, 3031 (1984).

3. (a) R. S. Mulliken, Phys. Rev. 50, 1017 (1936); (b) 51, 310 (1937); (c) 47, 413(1935); (d) J. Chem. Phys. 8, 382 (1940).

4. R. A. Boschi and D. R. Salahub, Mol. Phys. 24, 289 (1972).

5. T. K. Minton, G. M. Nathanson and Y. T. Lee, to be published in J. Chem. Phys.

6. We have checked the influence of saturation on $\beta$ by incorporating a saturation model $^{7}$ into our data analysis program, and we have found that for $\sigma F$ (cross section $\times$ laser intensity) of $\sim 0.1$, which is an upper limit for our experiment, the anisotropy parameter calculated without taking into account saturation would be $<0.1$ too low. However, as we did include the effect of saturation in our analysis and as the error in $\beta$ is greater than 0.1 due to the uncertainty in the fit to the data, saturation will in no way affect our results. 
7. (a) J. H. Ling and K. R. Wilson, J. Chem. Phys. 65, 881 (1976);(b) P. M. Kroger, P. C. Demou and S. J. Riley, J. Chem. Phys. 65, 1823 (1976).

8. G. E. Busch and K. R. Wilson, J. Chem. Phys. 56, 3638 (1972).

9. S. Riley and K. R. Wilson, Discuss. Faraday Soc. 53, 132 (1972).

10. G. N. A. van Veen, T. Baller, A. E. Devries and N. J. A. van Veen, Chem. Phys. 87, 405 (1984).

11. M. D. Barry and P. A. Gorry, Mol. Phys. 52, 461 (1984).

12. L. J. Butler, E. J. Hintsa, S. F. Shane and Y. T. Lee, J. Chem. Phys, to be published. 